\title{
Litz Wire Losses: Effects of Twisting Imperfections
}

\section{Conference Paper}

Author(s):

Guillod, Thomas; Huber, Jonas; Krismer, Florian; Kolar, Johann W. (D)

Publication date:

2017

Permanent link:

https://doi.org/10.3929/ethz-b-000187502

Rights / license:

In Copyright - Non-Commercial Use Permitted

Originally published in:

https://doi.org/10.1109/COMPEL.2017.8013327 


\title{
Litz Wire Losses: Effects of Twisting Imperfections
}

\author{
Power Electronic Systems Laboratory (PES) \\ ETH Zurich \\ 8092 Zurich, Switzerland
}

Thomas Guillod, Jonas Huber, Florian Krismer, and Johann W. Kolar

\begin{abstract}
High-Frequency (HF) litz wires are extensively used for the windings of Medium-Frequency (MF) magnetic components in order to reduce the impact of eddy current losses that originate from skin and proximity effects. Literature documents different methods to calculate eddy current losses in HF litz wires, however, most of the computation methods rely on perfect twisting of the strands, which is often not present in practice. This paper analyzes the implications of imperfect twisting on the current distribution among the different strands of HF litz wires and the corresponding losses by means of a fast 2.5D PEEC (Partial Element Equivalent Circuit) method. The effects of different types of twisting imperfections (at the bundle-, subbundle-, or strand-level) are examined. It is found that imperfect twisting can lead to increased losses (more than $100 \%$ ). However, perfect twisting of the strands, which is difficult to achieve, is often not required, i.e. suboptimal twisting is sufficient. Analytical expressions are given for distinguishing between critical and uncritical imperfections. The experimental results, conducted with a $7.5 \mathrm{kHz} / 65 \mathrm{~kW}$ transformer, reveal a reduction of the error on the predicted losses from $52 \%$ (ideal HF litz wire model) to $8 \%$ (presented model) and, thus, confirm the accuracy improvement achieved with the proposed approach.
\end{abstract}

Index Terms-Power Electronics, Litz Wires, Skin Effect, Proximity Effect, Twisting Imperfections, Medium-Frequency, Transformers, Inductors, PEEC, Numerical Simulations.

\section{INTRODUCTION}

The computation of winding losses represents an essential part of the modeling process of inductors and transformers. Power electronic converters are usually operating at Medium-Frequency (MF), which can be defined with the power/frequency product (usually $0.1-1.0 \mathrm{GHzW}$ for medium-power systems). At MF, the impacts of eddy current losses cannot be neglected any more. Usually, eddy current losses are split up into losses due to skin and proximity effects: the skin effect describes the eddy currents due to the magnetic field created by the current in the conductor itself and the proximity effect characterizes eddy currents that originate from an external magnetic field.

In order to obtain efficient MF inductors and transformers, the impacts of losses due to skin and proximity effects need to be mitigated. This is usually achieved with foil conductors or High-Frequency (HF) stranded conductors, e.g. Roebel bars or HF litz wires. In this regard, HF litz wires are very popular since they allow for low losses and great flexibility in the design of the magnetic component [1]-[5].

HF litz wires are composed of multiple insulated strands, as shown in Fig. 1. Each single strand is subject to skin and proximity effects and thus, the single strand diameter is selected with regard to acceptable losses [6]. The strands are twisted together in both radial and azimuthal directions:

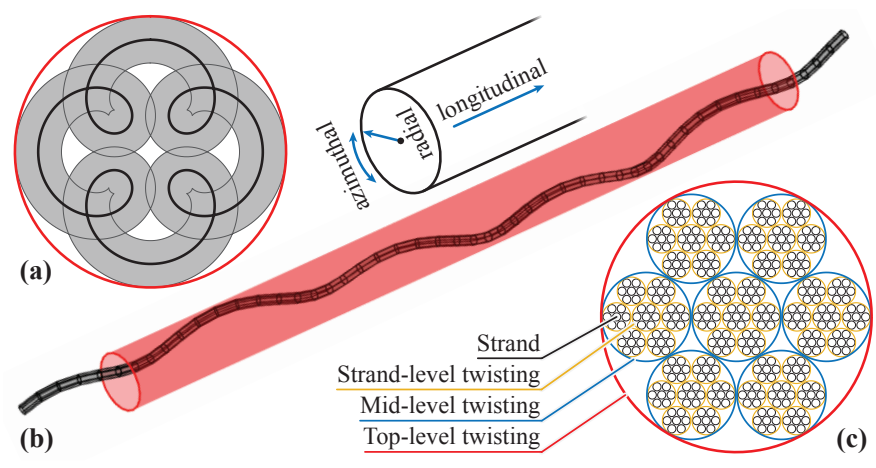

Fig. 1. (a) Projection and (b) 3D view of the twisting scheme (azimuthal and radial) of a single strand. The depicted path depends on the structure of the bundle, the pitch lengths of the bundles, and the used twisting scheme. (c) Packing structure of a HF litz wire with bundles and sub-bundles.

- The permutation in radial direction prevents eddy currents in case of an azimuthal magnetic field. An azimuthal field is created by the currents that are present in the considered HF litz wire itself.

- The permutation in azimuthal direction suppress the oriented area spanned between the strands with respect to an homogeneous external field. The cancellation of the magnetic flux between the strands, prevents the formation of circulating eddy currents.

In addition to the number of strands and the diameter of a single strand, the pitch (or length of lay), which is the spatial period length of the twisting (in longitudinal direction), denotes a third important parameter of a HF litz wire.

For HF litz wires with a high number of strands, simultaneous azimuthal and radial twisting is difficult to achieve. Such HF litz wires are often constructed in a recursive manner, i.e., the total HF litz wire is composed of twisted bundles of HF litz wires and each bundle itself can again be composed of twisted bundles or single strands. In this paper, the first twisting level (twisting of the strands) and the last level (forming the complete HF litz wire) are called strand-level and top-level twisting, respectively. Additional twisting levels between the top- and the strand-level are denoted as mid-levels, cf. Fig. 1(c).

With high numbers of strands and complex twisting schemes, the accurate computation of losses in such stranded conductors is a challenging task. Over the years, different calculation methods have been proposed for the calculation of the losses, which rely on analytical and/or numerical calculations [6]-[11]. However, it is found that the 
measured losses are often larger than the values computed with the aforementioned methods [1], [2], [12]. Different effects can explain such deviations:

- The (external) magnetic field is inaccurately computed [13]. Moreover, certain computational methods presuppose the external magnetic field to be homogeneous across the cross section of the HF litz wire [11].

- The terminations of the HF litz wire are generating losses. Moreover, terminations can add an impedance mismatch between the strands which leads to undesired circulating currents [2], [12], [14].

- Due to twisting, the length of the strands is greater than the length of the HF litz wire [15]. For typical HF litz wire, however, this effect is often negligible (less than $10 \%)$.

- The packing (hexagonal, square, etc.) of the strands is not homogeneous and/or not perfectly modeled [8], [9]. The exact packing in a HF litz wire, which is often not clearly defined, has only a limited impact on the losses (less than $10 \%$ ).

- A non-integer number of pitches is used, leading to circulating currents inside the HF litz wire [7], [16]. Alternatively, local variations (along distances that are similar to the pitch length) of the external magnetic field also create additional losses [12]. Such local variations are typically produced by the fringing fields of air gaps.

- An imperfect twisting scheme is used, leading to an inhomogeneous current distribution in the strands. This includes improper azimuthal and/or radial twisting (at the top-, strand-, or mid-level), poor choices of the ratios of the pitch lengths between twisting levels, etc.

The last two effects can lead to significant additional losses and commonly occur in practice. In particular, commercially available HF litz wires are frequently found to be subject to imperfect twisting [2]. Therefore, this paper analyzes the impact of the twisting scheme on the losses, which, to the knowledge of the authors, has not been studied in details in the literature. Section II summarizes loss computation methods for perfectly twisted HF litz wires. Section III analyzes the implications of twisting imperfections on the losses. Finally, Section IV, compares calculated results to experimental results, using a MF transformer that employs a HF litz wire with imperfect twisting.

\section{Perfect Twisting}

A perfectly twisted (radial and azimuthal directions) HF litz wire with an infinitesimally small pitch length is considered (ideal HF litz wire). The HF litz wire conducts the current $I_{\text {wire }}$ and is subject to a homogeneous external magnetic field, $H_{\text {ext }}$. Fig. 2 depicts the magnetic field determined with FEM simulations for a $343 \times 100 \mu$ m HF litz wire at different frequencies. At $1 \mathrm{MHz}$, the eddy currents generate losses but have a limited impact on the magnetic field distribution. For $f \gg 1 \mathrm{MHz}$ the skin depth is less than the strand diameter of a single strand and the eddy currents alter the magnetic field distribution, leading to a shielding

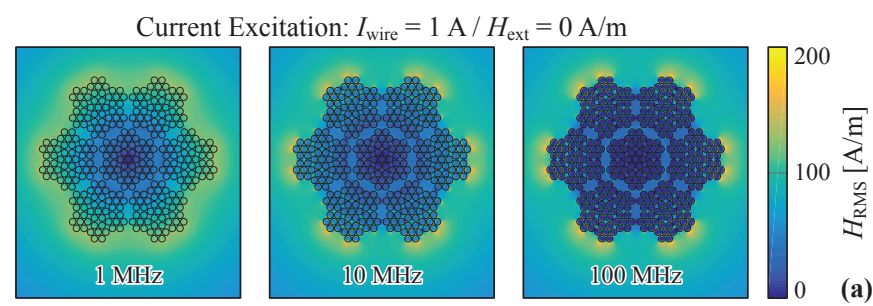

Field Excitation: $I_{\text {wire }}=0 \mathrm{~A} / H_{\mathrm{ext}}=1 \mathrm{~A} / \mathrm{m}$

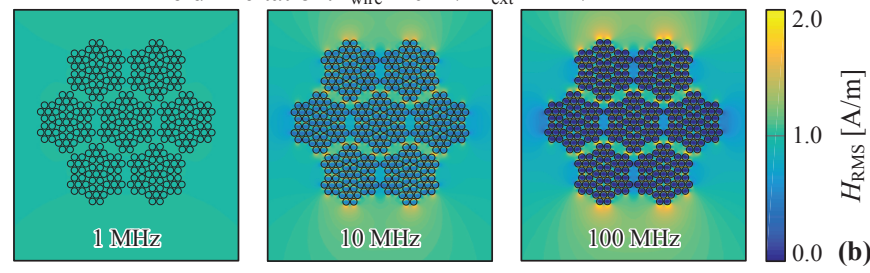

Fig. 2. (a) Low frequency and high frequency magnetic field patterns for current and (b) external field excitation. A $343 \times 100 \mu \mathrm{m}$ HF litz wire is considered $(2.5 \mathrm{~mm}$ outer diameter).

effect inside the strands. The corresponding losses, $P$, are expressed as [11]

$$
P(f)=R_{\mathrm{DC}}\left(K_{I}(f) I_{\text {wire }}^{2}+K_{H}(f) H_{\text {ext }}^{2}\right),
$$

where $K_{I}$ and $K_{H}$ are frequency-dependent parameters and $R_{\mathrm{DC}}$ denotes the low frequency resistance. The losses can be split up into four categories:

- DC losses (represented by $R_{\mathrm{DC}}$ ): conduction losses which are not linked to eddy currents.

- Skin effect (included in $K_{I}$ ): eddy current losses in a strand that originate from the field created by the current in the same strand.

- Internal proximity effect (included in $K_{I}$ ): eddy current losses in a strand that originate from the field created by the currents in the other strands.

- External proximity effect (included in $K_{H}$ ): eddy current losses in a strand by reason of an externally applied homogeneous magnetic field.

The parameters $R_{\mathrm{DC}}, K_{I}$, and $K_{H}$ are figures of merit for the HF litz wire itself. In a magnetic component (e.g. inductor, transformer), the relevant part of the external magnetic field, $H_{\mathrm{ext}}$, depends on the conductor current, $I_{\text {wire }}$, and the geometrical properties of the component (e.g. selected core, air-gap length, number of turns). This allows the definition of the equivalent $\mathrm{AC}$ resistance, which is a figure of merit for the complete winding of a defined magnetic component and not only for the HF litz wire. With (1), the equivalent AC resistance can be calculated according to

$$
P=R_{\mathrm{AC}}(f) I_{\text {wire }}^{2}, \quad H_{\mathrm{ext}} \sim I_{\text {wire }} .
$$

Different methods exist for the computation of the losses, which feature different hypotheses, high frequency accuracies, and computational costs. The most common methods are listed below:

- Asymptotic approximation (“Asymp”): The losses by reason of skin effect are neglected (the skin depth is assumed to be larger than the diameter of strands) 
and the losses due to proximity effects (internal and external) are modeled with a low-frequency asymptotic approximation. The packing pattern is only modeled with the fill factor [2], [6], [10].

- Bessel functions ("Bessel fct."): The losses due to skin and proximity effects are modeled with Bessel functions. HF effects are considered for every strand but the shielding effect of the eddy currents (cf. Fig. 2) is not modeled. The packing pattern is only modeled with the fill factor [2], [11].

- Biot-Savart discrete modeling ("Biot-Savart"): The losses by reason of skin and proximity effects are modeled with Bessel functions. The exact packing pattern is modeled and the magnetic field is computed with a Biot-Savart summation [5].

- FEM homogenization ("FEM hom."): The packing pattern of the HF litz wire is assumed to be periodic. A unit cell of the pattern is solved with FEM simulation and virtual material parameters (conductivity and permeability) are extracted for the packing pattern. Subsequently, the virtual conductor parameters are used to compute the losses. All high frequency effects are modeled. The limitations of this approach are the presupposed periodicity of the packing pattern and inaccuracies at the boundary of the HF litz wire [8], [9].

- FEM discrete modeling ("FEM disc."): All strands are modeled with FEM. The obtained solution contains all high frequency effects and considers the exact packing pattern [14]. Alternatively the PEEC (Partial Element Equivalent Circuit) method can be used [14].

Fig. 3 depicts a comparison between the methods for a $343 \times 100 \mu \mathrm{m}$ HF litz wire (cf. Fig. 2). The corner frequency $f_{\mathrm{c}, \mathrm{HF}}$ characterizes the maximum frequency up to which the low frequency asymptote of the proximity losses is considered to be valid and can be expressed as

$$
f_{\mathrm{c}, \mathrm{HF}}\left(d_{\mathrm{s}}\right) \approx \frac{32^{\frac{2}{3}}}{\pi \sigma \mu_{0} d_{\mathrm{s}}^{2}},
$$

where $\sigma$ is the conductivity and $d_{\mathrm{s}}$ the diameter of the strands. For $f<f_{\mathrm{c}, \mathrm{HF}}$, it can be concluded that the simplest method, the asymptotic approximation, features sufficient accuracy for most applications. For $f>f_{\mathrm{c}, \mathrm{HF}}$, the solutions based on Bessel functions or, if higher accuracy is required, the FEM-based methods need to be considered (cf. Fig. 2). Most commonly, however, $f>f_{\mathrm{c}, \mathrm{HF}}$ is avoided in order to avoid excessive HF losses and a HF litz wire with a smaller single strand diameter is considered [2]. Therefore, in this paper, $f<f_{\mathrm{c}, \mathrm{HF}}$ is presupposed. Furthermore, the comparative evaluation given in Fig. 3 reveals that an exact model of the packing pattern is not required.

For $f<f_{\mathrm{c}, \mathrm{HF}}$ the results obtained with all considered computational methods are matching. This indicates that potential deviations between computed and measured losses originate from phenomena that violate the hypothesis of a perfectly twisted HF litz wire with an infinitesimally small pitch length.
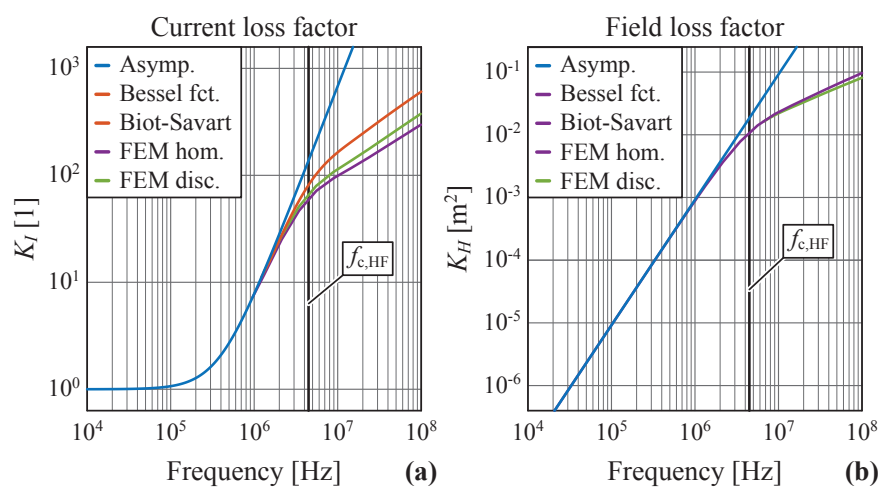

Fig. 3. (a) Loss factor $K_{I}$ and (b) $K_{H}$ computed with different methods (asymptotic approximation, Bessel functions, Biot-Savart summation, FEM homogenization, and FEM discrete modeling). A $343 \times 100 \mu \mathrm{m}$ HF litz wire is considered ( $2.5 \mathrm{~mm}$ outer diameter).

\section{TWISTING IMPERFECTIONS}

\section{A. Computation Method}

This section investigates the implications of different twisting schemes (e.g. radial, azimuthal, combined) and numbers of pitches (integer or non-integer) on the expected AC losses of HF litz wires. In such HF litz wires, the assumption of equal current distribution among the strands is, in general, incorrect and the aforementioned methods are not applicable.

In the literature, an analytical method for the simulation of imperfect HF litz wires has been proposed [16], especially with regard to the impact of the pitches. However, this method, which does not compute the current sharing between the strands, cannot be used for simulating complex twisting schemes. Further references propose 3D simulation methods, based on integral equations (e.g. PEEC), differential equations (e.g. FEM) [7], [17], [18], or both [13]. However, 3D methods are complex to implement and demand for very high computational cost, which limit the number of strands to less than hundred [7], [18]. For larger HF litz wires, massive parallel computing is required [17]. Furthermore, the 3D methods require a complete parametric representation of all the strands, which is difficult to obtain for HF litz wires with a high number of strands (e.g. consideration of deformations of the bundles) [10], [19].

The 2.5D PEEC method proposed here and briefly described in the following offers a trade-off between modeling accuracy, modeling effort, and computational cost. Fig. 4 presents the main steps of the method, which is based on a custom-built PEEC solver. First, the geometry (packing and twisting) of the HF litz wire is generated and the HF litz wire is sliced into different sections (about ten per pitch). In a second step, the inductance and resistance matrices are calculated using a $2 \mathrm{D}$ model. For taking the twisting scheme into account, the matrices are permuted (according to the slicing of the HF litz wire) and the total impedance matrix of the HF litz wire is generated. The current sharing problem is solved by equalizing the voltage drops (computed with the impedance matrix) between the strands [19]. Finally, the 


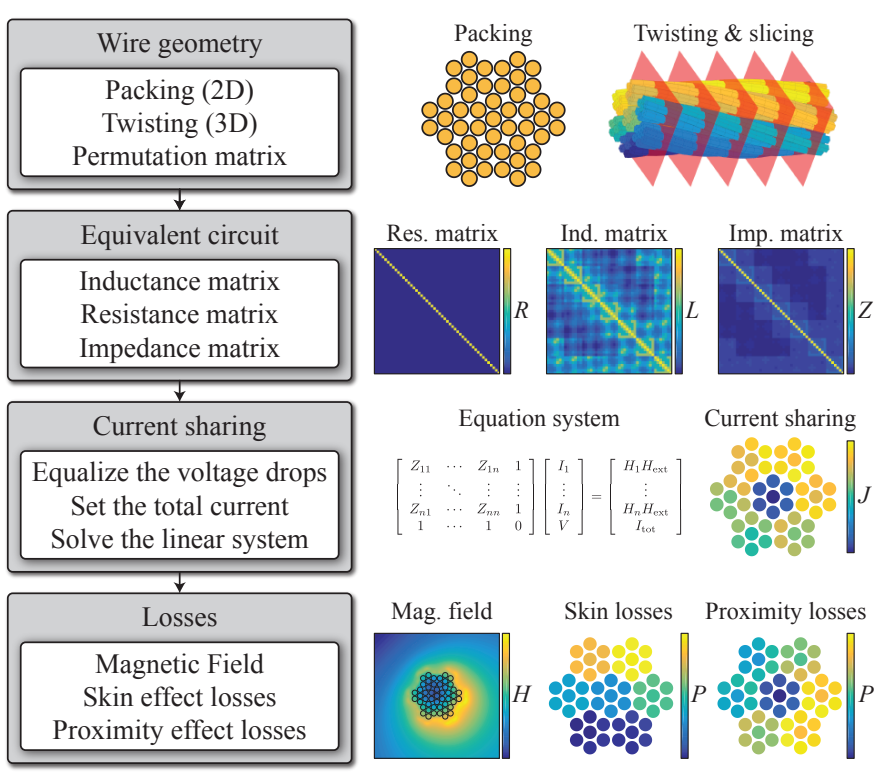

Fig. 4. Workflow for the computation of losses in HF litz wires with arbitrary twisting schemes and numbers of pitches. The analysis is based on a 2.5D PEEC method.

magnetic field and the losses (due to skin and proximity effects) are extracted.

Compared to a full 3D method, the 2.5D PEEC method neglects the current flows in azimuthal and radial directions, which are, however, much smaller than the longitudinal currents [19]. This implies that the method is not able to simulate configurations with a large external field applied in longitudinal direction, which are, anyway, uncommon for magnetic components. The main advantage of the 2.5D PEEC method is the reduced computational cost (and memory requirements), which enables the simulation of a HF litz wire with thousands of strands in less than a minutes on a personal computer. Moreover, the method does not require a complete 3D full parametric representation of the HF litz wire and, hence, substantially reduces the modeling complexity. Finally, the fact that the PEEC method is based on an equivalent circuit allows the combination of the HF litz wire model with external models, such as terminations [2], [14]. Furthermore, the method is also applicable to high frequencies $\left(f>f_{\mathrm{c}, \mathrm{HF}}\right)$, even if this case is not considered in this paper.

\section{B. Considered Twisting Imperfections}

Many different twisting schemes exist for HF litz wires [6], [14], [18], featuring different bundle structures, radial and/or azimuthal permutations, etc. However, most litz wires are constructed with the following twisting schemes:

- Perfect twisting ("PT"): the strands are perfectly twisted (radial and azimuthal direction). This ideal construction is difficult to realize with a large number of strands (more than six).

- Bunched wires ("BW"): the strands are only twisted in azimuthal direction. The strands located in the center of the wire, stay in the center and are not twisted. This represents the easiest and cheapest twisting scheme

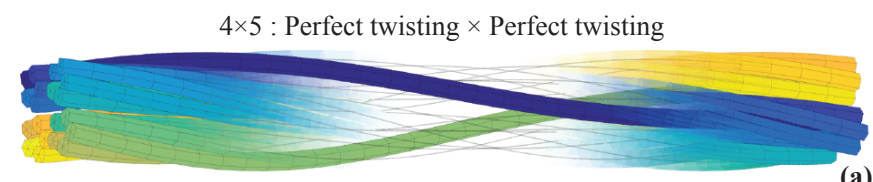

(a)

42 : Bunched wires

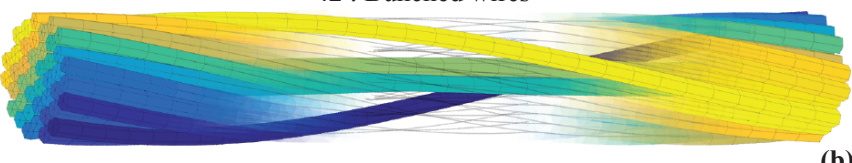

$7 \times 7:$ Bunched wires $\times$ Bunched wires
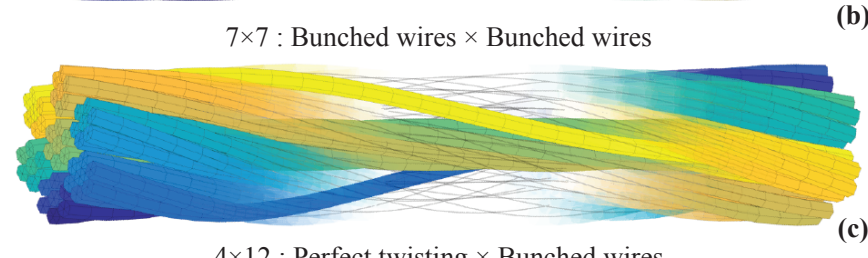

$4 \times 12$ : Perfect twisting $\times$ Bunched wires

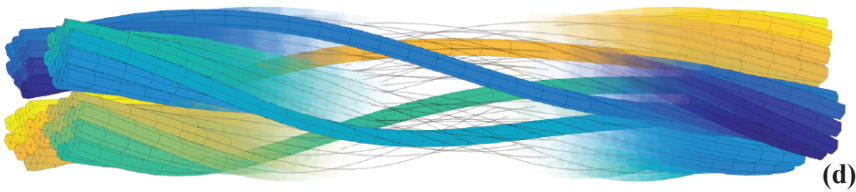

Fig. 5. (a) Perfect twisting, (b) bunched wires, (c) bundle in the center, and (d) hybrid twisting (perfect and bunched). Each title line specifies the corresponding number of bundles and the number of strands per bundle.

TABLE I

TWISTING SCHEMES $(343 \times 100 \mu \mathrm{m})$.

\begin{tabular}{lll}
\hline Name & Twisting scheme & Number of wires \\
\hline "343/PT/PT/PT" & PT $\times$ PT $\times$ PT & $7 \times 7 \times 7$ \\
“343/PT/PT/BW" & PT $\times$ PT $\times$ BW & $7 \times 7 \times 7$ \\
“343/PT/BW/BW" & PT $\times$ BW $\times$ BW & $7 \times 7 \times 7$ \\
“343/BW/BW/BW" & BW $\times$ BW $\times$ BW & $7 \times 7 \times 7$ \\
"343/PT/BW" & PT $\times$ BW & $7 \times 49$ \\
"343/BW" & BW & 343 \\
\hline
\end{tabular}

and is commonly found in commercially available HF litz wires [2], [6], [12].

These twisting schemes ("PT" and "BW") feature correct twisting in azimuthal direction since imperfect azimuthal twisting is uncommon for HF litz wires. Fig. 5 illustrates different combinations of the aforementioned twisting schemes (many levels of twisting). Fig. 5(c) shows an imperfect twisting scheme which feature an untwisted bundle in the center of the wire [2]. Fig. 5(d) depicts a hybrid twisting scheme, which is also commonly found: the "BW" twisting scheme is used at the strand-level and all other levels (e.g. top-level) employ perfect twisting ("PT") [6], [12].

All simulations consider the HF litz wire introduced in Section II $(343 \times 100 \mu \mathrm{m})$ and the six different combinations of the aforementioned twisting schemes ("PT", "BW", cf. Fig. 5) listed in Tab. I. The pitch length of the top-level twisting is $30 \mathrm{~mm}$. The ratio of the pitch between two successive twisting levels is set to 2.0 (given that the strand-level twisting pitch is the shortest).

\section{Simulation: Twisting Schemes}

The six twisting schemes listed in Tab. I are considered with an integer number of pitches. Fig. 6(a) illustrates the 

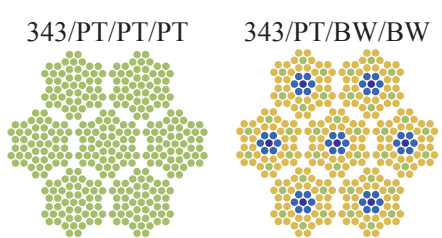

343/PT/PT/BW
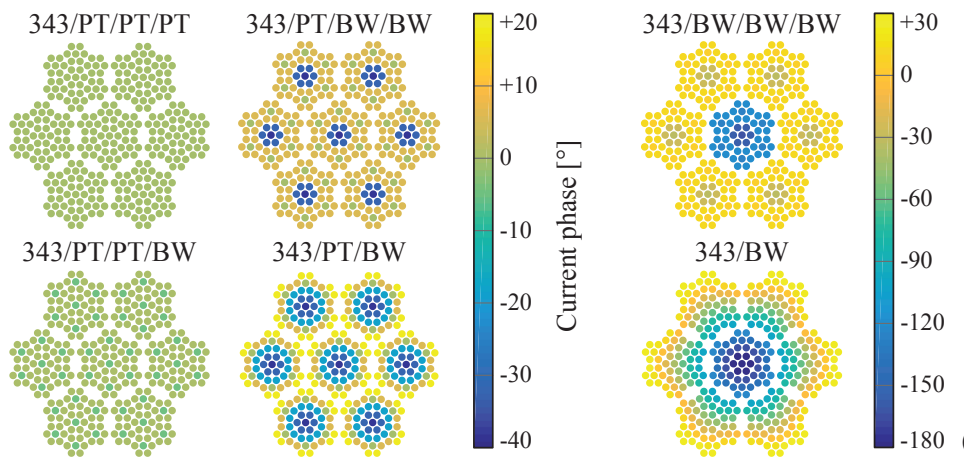

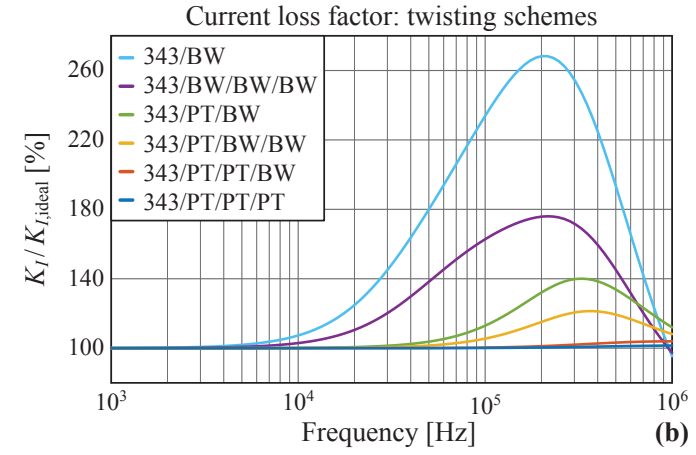

Fig. 6. (a) Phase differences of the strand currents (with respect to the phase of the total current, $I_{\text {wire }}$ ) at $100 \mathrm{kHz}$ for different twisting schemes $\left(I_{\text {wire }}=1 \mathrm{~A}, H_{\text {ext }}=0 \mathrm{~A} / \mathrm{m}\right.$ ). (b) Factor $K_{I}$ (cf. (1)) for different twisting imperfections (cf. Tab. I), compared to a perfectly twisted HF litz wire (cf. Fig. 3). A $343 \times 100 \mu \mathrm{m}$ HF litz wire is considered $(2.5 \mathrm{~mm}$ outer diameter).

current sharing for the case of a current excitation (no external field excitation). The current in the bundles which are not perfectly radially twisted is phase-shifted with respect to the total current. This implies that more current is flowing in the other bundles. This leads to increased losses according to Fig. 6(b) for $K_{I}$ (cf. (1)). The effect of the twisting on the DC resistance is small (less than $5 \%$ ) and, therefore, is neglected.

As expected, the loss increases are particularly pronounced (more than $100 \%$ ) for twisting imperfections at the toplevel ("343/BW/BW/BW", "343/BW"). For imperfect twisting at the mid-level ("343/PT/BW/BW" and "343/PT/BW"), the increase is already much smaller (less than $40 \%$ ). A twisting imperfection at the strand-level ("343/PT/PT/BW") has a negligible impact on the losses, showing that a perfect twisting is actually not required. Finally, the ideal twisting scheme ("343/PT/PT/PT") matches perfectly with the methods presented in Section II. At higher frequencies ( $f \approx 1 \mathrm{MHz}$, cf. Fig. 6(b)), the effects of twisting imperfections disappear and imperfectly twisted HF litz wires feature even lower losses, due better current distributions with respect to the high-frequency proximity losses [6], [11], [12].

The impact of the twisting schemes on the external field factor $K_{H}$ (cf. (1)) is negligible since the considered HF litz wires are correctly twisted in azimuthal direction and an integer number of pitches is chosen.

\section{Simulation: Number of Pitches}

The losses are further affected by the number of pitches (number of twisting periods) of the HF litz wire. For the sake of simplicity and brevity, only the twisting scheme " $343 / \mathrm{BW}$ " is considered. However, the results are similar for the remaining five schemes since the impacts of the pitch lengths of the sub-bundles (e.g. mid- and strand-level twisting) are less critical [16].

If a non-integer number of pitches is used, the impedance asymmetry between the strands is leading to circulating currents, as shown in Fig. 7(a) for 5.5 pitches and an external magnetic field excitation (no current excitation). Fig. 7(b) illustrates the impact of the number of pitches on
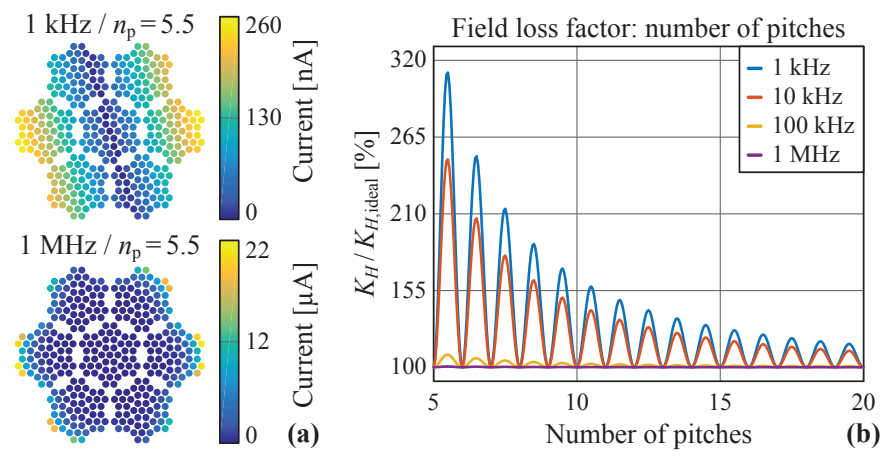

Fig. 7. (a) Amplitude of the strand currents for the twisting scheme "343/BW" with 5.5 pitches $\left(H_{\text {ext }}=1 \mathrm{~A} / \mathrm{m}, I_{\text {wire }}=0 \mathrm{~A}\right)$. (b) Factor $K_{H}$ (cf. (1)) for the twisting scheme "343/BW" and for different number of pitches compared to a perfectly twisted HF litz wire (cf. Fig. 3). A $343 \times 100 \mu \mathrm{m} \mathrm{HF}$ litz wire is considered $(2.5 \mathrm{~mm}$ outer diameter).

the external field factor $K_{H}$ (cf. (1)). The impact on $K_{I}$ is much smaller and therefore not considered.

The loss increase is particularly pronounced (more than $100 \%$ ) for short HF litz wires which is critical for windings with reduced number of turns. As expected, the impacts of non-integer numbers of pitches reduce significantly for increased lengths of the HF litz wire. At higher frequencies, the spatial distributions of the circulating currents change (cf. Fig. 7(a)), which leads to a mitigation of the losses due to the circulating currents between the strands [11]. By contrast, as shown in Fig. 3, the factor $K_{H}$ of an ideal HF litz wire increases rapidly over frequency. This implies that the effects of a non-integer number of pitches become negligible at higher frequencies.

The same effect appears for winding with an integer number of pitches placed in a non-homogeneous external magnetic field (variable in the longitudinal direction). Such local variations, which are typically produced by the fringing fields of air gaps, also lead to circulating currents, as shown in Fig. 7(a).

\section{E. Analytical Approximations \& Design Recommendations}

Even though the 2.5D PEEC simulations are fast, analytical formulas are still required for a rapid assessment of the impacts of twisting imperfections on the losses. For 

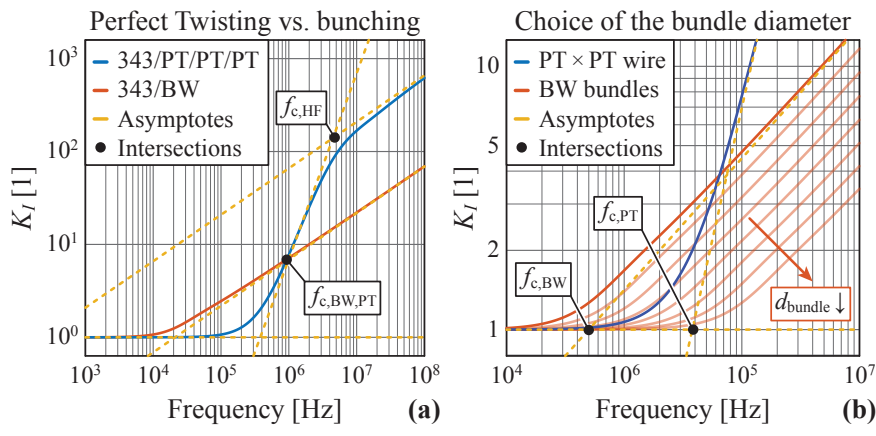

Fig. 8. (a) Loss factors, $K_{I}$, for the twisting schemes "343/PT/PT/PT" and "343/BW". (b) Loss factors, $K_{I}$, for the bunched bundles (diameter $d_{\text {bundle }}$ ) and the perfectly twisted HF litz wire (diameter $d_{\text {outer }}$ ). Different diameters are considered for $d_{\text {bundle }}$ (from $0.3 \mathrm{~mm}$ to $1.6 \mathrm{~mm}$ ). For both plots, the corresponding asymptotes and intersections are also shown. A $343 \times 100 \mu \mathrm{m}$ HF litz wire is considered $\left(d_{\text {outer }}=2.5 \mathrm{~mm}\right)$.

computing the loss increases due non-integer numbers of pitches or non-homogeneous external field, the analytical formulas presented in [16] can be used. For the evaluation of different twisting schemes (cf. Fig. 5), the following approach can be used.

First the twisting schemes "343/PT/PT/PT" and "343/BW", which represent the extreme cases (cf. Fig. 6), are considered. Fig. 8(a) depicts the corresponding loss factors, $K_{I}$, and presents the different asymptotes. With these asymptotes, an expression for the frequency, $f_{\mathrm{c}, \mathrm{BW}, \mathrm{PT}}$, where the two curves intersect, can be derived as

$$
f_{\mathrm{c}, \mathrm{BW}, \mathrm{PT}}\left(d_{\text {outer }}, d_{\mathrm{s}}\right) \approx \frac{1024^{\frac{1}{3}}}{\pi \sigma \mu_{0} F d_{\text {outer }}^{\frac{2}{3}} d_{\mathrm{s}}^{\frac{4}{3}}},
$$

where $\sigma$ is the conductivity and $d_{\mathrm{s}}$ the diameter of the strands, $d_{\text {outer }}$ the external HF litz wire diameter, and $F$ the fill factor. It can be seen that a bunching of the all strands would be preferable for $f>f_{\mathrm{c}, \mathrm{BW}, \mathrm{PT}}$, due to the superior high frequency properties [6]. However, HF litz wires are usually operated at $f<f_{\mathrm{c}, \mathrm{BW}, \mathrm{PT}}$ such that twisting in radial direction is also required [2], [6].

Radial twisting is difficult to achieve for large numbers of strands and a construction based on bundles with reduced numbers of single strands is preferred, instead. In case of HF litz wires with very high numbers of single strands, many levels of bundles are required, which makes the process of twisting difficult and expensive. According to the results depicted in Fig. 6, however, certain twisting imperfections can be acceptable. Therefore, an expression is required in order to assess the impacts of given imperfections on the expected losses.

Since imperfections in the top-most level are most critical and should be avoided (for $f<f_{\mathrm{c}, \mathrm{BW}, \mathrm{PT}}$ ), a HF litz wire with the twisting scheme "PT $\times$ BW" is selected and compared to a perfectly twisted HF litz wire ("PT $\times$ PT"). The diameter of each bundle is $d_{\text {bundle }}$ and the outer diameter of the HF litz wire is $d_{\text {outer }}$ Fig. 8(b) shows the loss factors, $K_{I}$, calculated for single bundles and the loss factor of the (complete) perfectly twisted HF litz wire. The corner frequencies for
TABLE II

$7.5 \mathrm{kHz} / 65 \mathrm{~kW}$ MF TRANSFORMER.

\begin{tabular}{lll}
\hline Name & HV winding & LV winding \\
\hline Voltage & $\pm 1.1 \mathrm{kV}$ & $\pm 0.8 \mathrm{kV}$ \\
RMS Current & $70 \mathrm{~A}$ & $95 \mathrm{~A}$ \\
Winding size & $6.0 \times 72 \mathrm{~mm}$ & $8.1 \times 70 \mathrm{~mm}$ \\
\hline Litz wire profile & Rectangular & Rectangular \\
Litz wire size & $6.0 \times 6.0 \mathrm{~mm}$ & $8.1 \times 8.1 \mathrm{~mm}$ \\
Number of strands & $700 \times 200 \mu \mathrm{m}$ & $1000 \times 200 \mu \mathrm{m}$ \\
Twisting Scheme & $\mathrm{BW} \times \mathrm{PT} \times \mathrm{BW}$ & $\mathrm{BW} \times \mathrm{BW} \times \mathrm{BW}$ \\
Number of wires & $7 \times 5 \times 20$ & $7 \times[10,11] \times 14$ \\
\hline
\end{tabular}

the perfectly twisted HF litz wire and the bundles, $f_{\mathrm{c}, \mathrm{PT}}$ and $f_{\mathrm{c}, \mathrm{BW}}$, can be expressed as:

$$
\begin{aligned}
f_{\mathrm{c}, \mathrm{PT}}\left(d_{\text {outer }}, d_{\mathrm{s}}\right) & \approx \frac{\sqrt{128}}{\pi \sigma \mu_{0} F d_{\text {outer }} d_{\mathrm{s}}}, \\
f_{\mathrm{c}, \mathrm{BW}}\left(d_{\text {bundle }}\right) & \approx \frac{16}{\pi \sigma \mu_{0} F d_{\text {bundle }}^{2}} .
\end{aligned}
$$

In order to avoid that the eddy current losses in the single bundles lead to a considerable increase of the overall losses, the corner frequency of the bundles needs to be greater than the operating frequency (by approximately a factor of two/three). With this, the following condition for the diameter of each bundle is derived:

$$
d_{\text {bundle }}\left(f_{\mathrm{c}}\right)<\frac{4}{\sqrt{\pi \sigma \mu_{0} F f_{\mathrm{c}}}}, \quad f_{\mathrm{c}}<f_{\mathrm{c}, \mathrm{BW}}<f_{\mathrm{c}, \mathrm{PT}} .
$$

where $f_{\mathrm{c}}$ is the selected corner frequency. These results can also be generalized to HF litz wires with arbitrary numbers of twisting levels. The diameters of the largest bundles resulting from an imperfect twisting scheme (e.g. "BW") should not exceed the value computed with (7).

For the construction of a HF litz wire with a given outer diameter $\left(d_{\text {outer }}\right)$ and strand diameter $\left(d_{\mathrm{s}}\right)$, the following design procedure can be used (for $f<f_{\mathrm{c}, \mathrm{BW}, \mathrm{PT} \text { ): }}$

- The bundles diameter ( $d_{\text {bundle }}$, cf. (7)) is computed for the strand-level twisting. This strand-level bundle is composed of bunched strands. Using bundles with a smaller diameter only marginally reduces the losses but significantly increase the complexity of the twisting scheme and potentially reduce the fill factor.

- From the diameters $d_{\text {outer }}$ and $d_{\text {bundle }}$, the number of bundle is extracted.

- If the number of bundles is too large for a "PT $\times$ BW" twisting scheme additional mid-level twisting steps should be added (e.g. "PT $\times \mathrm{PT} \times \mathrm{BW}$ ').

Now it is possible to combine the aforementioned effects (e.g. non ideal twisting, number of pitches) into an accurate model of the windings of a real component.

\section{Measurements of Twisting Imperfections}

A $7.5 \mathrm{kHz} / 65 \mathrm{~kW}$ MF transformer is considered, cf. Fig. 10. This transformer is used in a DC-DC converter composing a Solid-State Transformer (SST) [20], [21]. Tab. II summarizes the relevant parameters of the windings and the employed HF litz wires. All measurements are conducted with the 

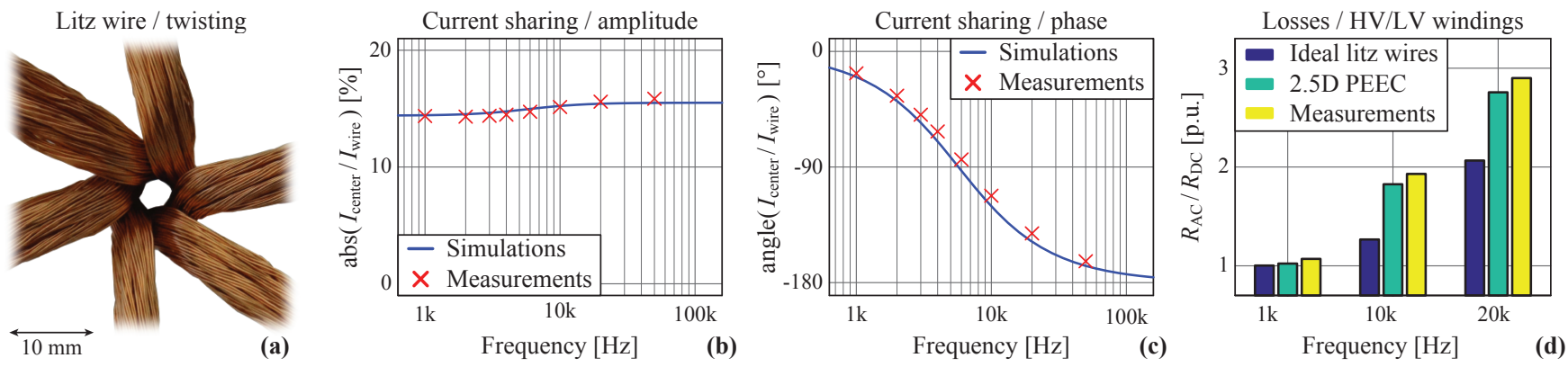

Fig. 9. (a) HF litz wire structure for the HV winding where the untwisted bundle in the center has been removed. (b) Amplitude and (c) phase of the current in the center bundle compared to the total current in the HV winding. (d) Ratio $R_{\mathrm{AC}} / R_{\mathrm{DC}}$ (cf. (2), for both windings). Also shown are the simulated losses for hypothetical perfectly twisted HF litz wires and for the actually present imperfect twisting.
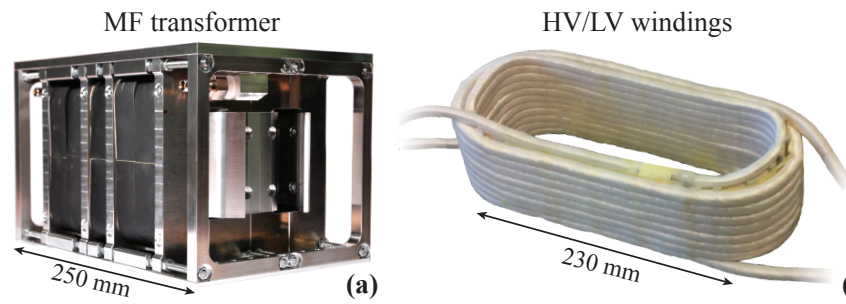

(b)

Fig. 10. (a) Considered $7.5 \mathrm{kHz} / 65 \mathrm{~kW}$ MF transformer. (b) HV/LV windings without the magnetic cores.

magnetic (nanocrystalline) cores being installed in order to obtain a realistic distribution of the magnetic field. The simulations employ the method presented in Fig. 4 and the external magnetic field is calculated with FEM. The number of pitches is very large (more than 70 for each winding), hence, an integer number of pitches has been assumed in the simulations.

In a first step, the current distribution among the bundles of the HV winding has been measured at different frequencies, with a short-circuit being applied to the LV winding. Fig. 9(a) shows the structure of the HF litz wire of the HV winding, for which an untwisted bundle is present in the center ("BW" at the top-level twisting, cf. Fig. 5(c)). This center bundle has been removed in Fig. 9(a) to highlight the present construction of the HF litz wire. Therefore, an unequal current distribution among the bundles is expected (cf. Fig. 6 and (7)). Figs. 9(b)-(c) depict the ratio of the currents in the center bundle to the total current, with respect to amplitude and phase. A good agreement between simulations and measurements is achieved. Furthermore, it appears that measuring the amplitude of the current in the center bundle is insufficient since almost only the phase is affected by the imperfect twisting. Furthermore, it is found that the presence of the magnetic cores has only a minor impact on the current distribution in the HF litz wire (in both, simulations and measurements).

In a second step, the winding losses have been measured with a "Yokogawa 3000WT" power analyzer (LV winding short-circuited). In the course of this measurement, the ratio $R_{\mathrm{AC}} / R_{\mathrm{DC}}$ is determined, since it is a figure of merit for the losses of the windings (for both HV and LV, cf. (2)). Fig. 9(d) presents a comparative evaluation of the results for the simulations and the measurements conducted for the present HF litz wires and, in addition, includes the simulation results for hypothetical perfectly twisted HF litz wires. At $10 \mathrm{kHz}$ (near the operating frequency), the resistance ratio for hypothetical perfectly twisted HF litz wires is 1.27 , the simulated ratio for the non-ideal HF litz wires is 1.79 , and the measurement returns 1.93 . Hence, the mismatch between expected losses with perfectly twisted HF litz wires and the measurements (52\%) can be explained with the presented simulation method.

\section{CONCLUSION}

This paper investigates the implications of imperfections in HF litz wires (e.g. number of pitches, twisting) on the losses and particularly focuses on imperfect twisting which is commonly observed in commercially available HF litz wires. A fast 2.5D PEEC solver, which is able to simulate HF litz wires with thousands of strands, has been implemented to simulate the current distribution among the strands and determine the losses.

According to the results obtained from the analysis of different types of imperfect twisting schemes it is found that imperfect twisting, in particular of the top-level bundles, can lead to a significant increase of losses due to eddy currents (up to $100 \%$ increase). It is also found that imperfections may be tolerated at sub-bundle-levels. In this context, simple analytical expressions are derived and the results are summarized in a design guideline for the construction of suitable HF litz wires. Furthermore, an increase of the losses is to be expected for windings with low number of pitches and/or non-homogeneous external magnetic field.

The presented method is finally applied to a $7.5 \mathrm{kHz} / 65 \mathrm{~kW}$ MF transformer, which employs imperfectly twisted HF litz wires, for experimental verification. Both, the measured current distribution among the bundles and the losses, are in good agreement with the simulations. The imperfections of the HF litz wires lead to an increase of the winding losses by $52 \%$.

\section{ACKNOWLEDGMENT}

This work is part of the Swiss Competence Centers for Energy Research (SCCER) initiative which is supported by the Swiss Commission for Technology and Innovation (CTI). 


\section{REFERENCES}

[1] S. Zhao, Q. Li, and F. C. Lee, "High Frequency Transformer Design for Modular Power Conversion from Medium Voltage AC to $400 \mathrm{~V}$ DC," in Proc. of the IEEE Applied Power Electronics Conf. and Expo. (APEC), 2017, pp. 2894-2901.

[2] M. Leibl, G. Ortiz, and J. W. Kolar, "Design and Experimental Analysis of a Medium Frequency Transformer for Solid-State Transformer Applications," IEEE Trans. Emerg. Sel. Topics Power Electron., vol. 5 no. 1, pp. 110-123, 2017.

[3] F. Kieferndorf, U. Drofenik, F. Agostini, and F. Canales, "Modular PET, Two-Phase Air-Cooled Converter Cell Design and Performance Evaluation with 1.7kV IGBTs for MV Applications," in Proc. of the IEEE Applied Power Electronics Conf. and Expo. (APEC), 2016, pp. 472-479.

[4] T. Guillod, F. Krismer, and J. W. Kolar, "Electrical Shielding of MV/MF Transformers Subjected to High dv/dt PWM Voltages,” in Proc. of the IEEE Applied Power Electronics Conf. and Expo. (APEC), 2017, pp. 2502-2510.

[5] J. Acero, R. Alonso, J. M. Burdío, l. A. Barragán, and D. Puyal, "Frequency-Dependent Resistance in Litz-Wire Planar Windings for Domestic Induction Heating Appliances," IEEE Trans. Power Electron. vol. 21, no. 4, pp. 856-866, 2006.

[6] C. R. Sullivan and R. Y. Zhang, "Simplified Design Method for Litz Wire," in Proc. of the IEEE Applied Power Electronics Conf. and Expo. (APEC), 2014, pp. 2667-2674.

[7] R. Y. Zhang, J. K. White, J. G. Kassakian, and C. R. Sullivan, "Realistic Litz Wire Characterization using Fast Numerical Simulations," in Proc. of the IEEE Applied Power Electronics Conf. and Expo. (APEC), 2014 pp. $738-745$.

[8] D. C. Meeker, "An Improved Continuum Skin and Proximity Effect Model for Hexagonally Packed Wires,” Journal of Computational and Applied Mathematics, vol. 236, no. 18, pp. 4635-4644, 2012.

[9] N. Xi and C. R. Sullivan, "An Equivalent Complex Permeability Model for Litz-Wire Windings," IEEE Trans. Ind. Appl., vol. 45, no. 2, pp. 854-860, 2009.

[10] C. R. Sullivan, "Optimal Choice for Number of Strands in a Litz-Wire Transformer Winding," IEEE Trans. Power Electron., vol. 14, no. 2, pp. 283-291, 1999.
[11] H. Mühlethaler, "Modeling and Multi-Objective Optimization of Inductive Power Components,” Ph.D. dissertation, ETH Zurich, 2012.

[12] H. Rossmanith, M. Doebroenti, M. Albach, and D. Exner, "Measurement and Characterization of High Frequency Losses in Nonideal Litz Wires," IEEE Trans. Power Electron., vol. 26, no. 11, pp. 3386-3394, 2011.

[13] A. Rosskopf, E. Baer, C. Joffe, and C. Bonse, "Calculation of Power Losses in Litz Wire Systems by Coupling FEM and PEEC Method," IEEE Trans. Power Electron., vol. 31, no. 9, pp. 6442-6449, 2016.

[14] A. Rosskopf, E. Bar, and C. Joffe, "Influence of Inner Skin-and Proximity Effects on Conduction in Litz Wires," IEEE Trans. Power Electron., vol. 29, no. 10, pp. 5454-5461, 2014.

[15] X. Tang and C. R. Sullivan, "Stranded Wire with Uninsulated Strands as a Low-Cost Alternative to Litz Wire," in Proc. of the IEEE Power Electronics Specialist Conf. (PESC), vol. 1, 2003, pp. 289-295.

[16] C. R. Sullivan and R. Y. Zhang, "Analytical Model for Effects of Twisting on Litz-Wire Losses," in Proc. of the IEEE Control and Modeling for Power Electronics Conf. (COMPEL), 2014, pp. 1-10.

[17] R. Scapolan, "Modélisation Electromagnétique 3D d'Inducteurs Multibrins-Développement d'une Méthode Intégrale Parallélisée (in French)," Ph.D. dissertation, Université de Grenoble, 2014.

[18] P. B. Reddy, T. M. Jahns, and T. P. Bohn, "Transposition Effects on Bundle Proximity Losses in High-speed PM Machines," in Proc. of the IEEE Energy Conversion Congr. and Expo. (ECCE USA), 2009, pp. 1919-1926.

[19] G. Cerri, S. A. Kovyryalov, and V. M. Primiani, "Modelling of a Litz-Wire Planar Winding Geometry for an Accurate Reactance Evaluation," IET Science, Measurement \& Technology, vol. 4, no. 4, pp. 214-219, 2010.

[20] T. Guillod, J. E. Huber, G. Ortiz, A. De et al., "Characterization of the Voltage and Electric Field Stresses in Multi-Cell Solid-State Transformers," in Proc. of the IEEE Energy Conversion Congr. and Expo. (ECCE USA), 2014, pp. 4726-4734.

[21] J. E. Huber and J. W. Kolar, "Volume/Weight/Cost Comparison of a 1MVA $10 \mathrm{kV} / 400 \mathrm{~V}$ Solid-State Against a Conventional Low-Frequency Distribution Transformer," in Proc. of the IEEE Energy Conversion Congr. and Expo. (ECCE USA), 2014, pp. 4545-4552. 